\title{
A simple sufficient condition for the quasiconvexity of elastic stored-energy functions in spaces which allow for cavitation
}

\author{
Jonathan J. Bevan ${ }^{1}$ • Caterina Ida Zeppieri ${ }^{2}$
}

Received: 13 July 2015 / Accepted: 4 March 2016 / Published online: 30 March 2016

(C) The Author(s) 2016. This article is published with open access at Springerlink.com

\begin{abstract}
In this note we formulate a sufficient condition for the quasiconvexity at $x \mapsto$ $\lambda x$ of certain functionals $I(u)$ which model the stored-energy of elastic materials subject to a deformation $u$. The materials we consider may cavitate, and so we impose the wellknown technical condition (INV), due to Müller and Spector, on admissible deformations. Deformations obey the condition $u(x)=\lambda x$ whenever $x$ belongs to the boundary of the domain initially occupied by the material. In terms of the parameters of the models, our analysis provides an explicit $\lambda_{0}>0$ such that for every $\lambda \in\left(0, \lambda_{0}\right]$ it holds that $I(u) \geq I\left(u_{\lambda}\right)$ for all admissible $u$, where $u_{\lambda}$ is the linear map $x \mapsto \lambda x$ applied across the entire domain. This is the quasiconvexity condition referred to above.
\end{abstract}

Mathematics Subject Classification $49 \mathrm{~J} 40 \cdot 74 \mathrm{~B} 20$

\section{Introduction}

Since the seminal work of Ball [3], the phenomenon of cavitation in nonlinear elasticity has been studied by many authors, with significant advances $[9,10,15]$ having been made in the case that an appropriately defined surface energy be part of the cost of deforming a material. In this note we consider the original case of a purely bulk energy

Communicated by N. Trudinger.

Jonathan J. Bevan

j.bevan@surrey.ac.uk

Caterina Ida Zeppieri

caterina.zeppieri@uni-muenster.de

1 Department of Mathematics, University of Surrey, Guildford GU2 7XH, United Kingdom

2 Institut für Numerische und Angewandte Mathematik, Universität Münster, Einsteinstr. 62, 48149 Münster, Germany 


$$
I(u)=\int_{\Omega} W(\nabla u(x)) d x,
$$

where as usual $u: \Omega \subset \mathbb{R}^{n} \rightarrow \mathbb{R}^{n}$ represents a deformation of an elastic material occupying the domain $\Omega$ in a reference configuration, and where $n=2$ or $n=3$. Our goal is to give a straightforward, explicit characterization of those affine boundary conditions of the form

$$
u_{\lambda}(x):=\lambda x,
$$

where $\lambda$ is a positive parameter, which obey the quasiconvexity inequality ${ }^{1}$

$$
I(u) \geq I\left(u_{\lambda}\right) .
$$

In the case of radial mappings [3] it is this inequality which must be violated in order that a global minimizer of $I$ might cavitate (i.e., where a hole is created in the deformed material), a crucial ingredient of which is the application of a large enough stretch on $\partial \Omega$ (i.e., taking $\lambda$ sufficiently large). When deformations are not restricted to any particular type we are still interested in whether the quasiconvexity inequality holds for a given $\lambda$ since it rules out the possibility that a global energy minimizer cavitates. Thus the largest $\lambda$ for which (1.2) holds is sometimes referred to as a critical load. Our chief inspiration for this work is [14], where bounds for the critical load are given in terms of constants appearing in certain isoperimetric inequalities. We use a different technique to find an explicit lower bound on the critical load in the two and three dimensional settings. The main results in this direction are summarised in Theorems 2.10 and 3.5.

Our method also yields conditions on $\nabla u$ for the inequality (1.2) to be close to an equality in the sense that if $\delta(u):=I(u)-I\left(u_{\lambda}\right)$ is small and positive then, in the two dimensional case

$$
\int_{\Omega} \min \left\{|\nabla u-\lambda \mathbf{1}|^{2},|\nabla u-\lambda \mathbf{1}|^{q}\right\} d x \leq c \delta(u),
$$

where $1<q<2$ is an exponent governing the growth of the stored-energy function $W$ appearing in (1.1). See Theorem 2.11 for the latter. The corresponding condition in three dimensions is

$$
\int_{\Omega}|\nabla u-\lambda \mathbf{1}|^{q} d x \leq c \delta(u),
$$

where $2<q<3$ : see Theorem 3.6 for details. In both cases the Friesecke, James and Müller rigidity estimate [8, Theorem 3.1] (see also [5, Theorem 1.1]) is used in conjunction with the boundary condition to recover information apparently lost in deriving sufficient conditions for (1.2). We also note that these conditions are invariant under the elasticity scaling in which a function $v(x)$, say, is replaced ${ }^{2}$ by $v^{\epsilon}(x)=\frac{1}{\epsilon} v(\epsilon x)$, where $\epsilon>0$. This is important in view of the example in [17, Section 1]. The latter says, among other things, that, in the absence of surface energy, a deformation which cavitates at just one point in the material can have the same energy as another deformation with infinitely many cavities.

The setting we work in is motivated by [15] in the sense that we impose condition (INV), a topological condition which is explained later. Cavitation problems must be posed in function spaces containing discontinuous functions. In particular, Sobolev spaces of the form $W^{1, q}\left(\Omega, \mathbb{R}^{n}\right)$ with $q \geq n$ are not appropriate, since their members are necessarily continuous. In the case $q>n$ this follows from the Sobolev embedding theorem, while if $q=n$

\footnotetext{
1 Strictly speaking, this is a $W^{1, q}$-quasiconvexity inequality; the term quasiconvexity usually refers to the case in which $I(u) \geq I\left(u_{\lambda}\right)$ holds for all Lipschitz $u$ agreeing with $u_{\lambda}$ on $\partial \Omega$. See, e.g., [4] for the distinction.

2 This is an oversimplification: see [4, Proposition 2.3] or [17] for full details.
} 
then well-known results [18,19], applying to maps $u$ with $\operatorname{det} \nabla u>0$ a.e., imply that $u$ has a continuous representative. Thus we work in $W^{1, q}\left(\Omega, \mathbb{R}^{n}\right)$, where $n-1<q<n$, and in so doing we are able to take advantage of existing results, including but not only those of [15].

The stored-energy functions we consider in the two dimensional case have the form

$$
W(A):=|A|^{q}+h(\operatorname{det} A)
$$

where $1<q<2$ and where $h: \mathbb{R} \rightarrow[0,+\infty]$ satisfies

(H1) $h$ is convex and $C^{1}$ on $(0,+\infty)$;

(H2) $\lim _{t \rightarrow 0+} h(t)=+\infty$ and $\liminf _{t \rightarrow \infty} \frac{h(t)}{t}>0$;

(H3) $h(t)=+\infty$ if $t \leq 0$.

In three dimensions the appropriate class of $W$ is detailed in Sect. 3. In both cases we define a set of admissible deformations

$$
\mathcal{A}_{\lambda}:=\left\{u \in W^{1, q}\left(\Omega, \mathbb{R}^{n}\right): u=u_{\lambda} \text { on } \partial \Omega, \operatorname{det} \nabla u>0 \text { a.e. in } \Omega\right\} .
$$

It is made clear in [3] and [16] that when $\lambda$ is sufficiently large there are maps $u_{0}$ belonging to $\mathcal{A}_{\lambda}$ of the form

$$
u_{0}(x)=r(|x|) \frac{x}{|x|}
$$

with $r(0)>0$, such that

$$
I\left(u_{0}\right)<I\left(u_{\lambda}\right) .
$$

The growth of $h(t)$ for large values of $t$ is pivotal in ensuring that such an inequality can hold. Thus the integrand $W$ is not $\left(W^{1, q}\right.$-) quasiconvex at $\lambda \mathbf{1}$. The loss of quasiconvexity is typically associated with so-called cavitating maps like $u_{0}$, whose distributional Jacobian Det $\nabla u_{0}$ is proportional to a Dirac mass, a remark first made by Ball in [3].

For later use, we recall that the distributional Jacobian of a mapping in $W^{1, p}\left(\Omega, \mathbb{R}^{n}\right)$, with $p>n^{2} /(n+1)$, is defined by

$$
(\operatorname{Det} \nabla u)(\varphi)=-\frac{1}{n} \int_{\Omega} \nabla \varphi \cdot(\operatorname{adj} \nabla u) u d x,
$$

where $\varphi$ belongs to $C_{0}^{\infty}(\Omega)$. When $u$ is $C^{2}$ the distributional Jacobian coincides with the Jacobian det $\nabla u$. The same is true if, more generally, $u \in W^{1, p}(\Omega)$ with $p \geq n^{2} /(n+1)$ and Det $\nabla u$ is a function (see [11]).

The paper is arranged as follows: after a short explanation of notation, we consider the two and three dimensional cases separately in Sects. 2 and 3 respectively. Subsection 2.1 contains the bulk of the estimates needed for (1.3); the relevant estimates in the three dimensional case draw on these results and are presented succinctly in Sect. 3. Along the way, we give a slight improvement of [20, Lemma 2.15], and, as a byproduct of our work in three dimensions we are led to a conjecture concerning the quasiconvexity of a certain function which, to the best of our knowledge, has not yet been considered in the literature.

\subsection{Notation}

We denote the $n \times n$ real matrices by $\mathbb{R}^{n \times n}$ and the identity matrix by 1 . Throughout, $\Omega \subset \mathbb{R}^{n}$ is a fixed, bounded domain with Lipschitz boundary, $B(a, R)$ represents the open ball in $\mathbb{R}^{n}$ centred at $a$ with radius $R>0$ and $S(a, R):=\partial B(a, R)$. Other standard notation includes $\mathcal{L}^{n}$ for the Lebesgue measure in $\mathbb{R}^{n}$. 
The inner product of two matrices $A, B \in \mathbb{R}^{n \times n}$ is $A \cdot B:=\operatorname{tr}\left(A^{T} B\right)$. This obviously holds for vectors too. Accordingly, we make no distinction between the norm of a matrix and that of a vector: both are defined by $|v|:=(v \cdot v)^{\frac{1}{2}}$. For any $n \times n$ matrix we write $\operatorname{adj} A:=(\operatorname{cof} A)^{T}$, while $\operatorname{tr} A$ and $\operatorname{det} A$ denote, as usual, the trace and determinant of $A$, respectively. Other notation will be introduced when it is needed.

\section{The two dimensional case}

The relevance of the distributional Jacobian to the loss of quasiconvexity can be seen using the following argument, the first part of which is due originally to Ball [2]. Firstly, the convexity of $A \mapsto|A|^{q}$ and of $h$ implies that

$$
W(\nabla u) \geq W(\lambda \mathbf{1})+q|\lambda \mathbf{1}|^{q-2} \lambda \mathbf{1} \cdot(\nabla u-\lambda \mathbf{1})+h^{\prime}\left(\lambda^{2}\right)\left(\operatorname{det} \nabla u-\lambda^{2}\right),
$$

which, when $u \in \mathcal{A}_{\lambda}$, can be integrated over $\Omega$; the result is

$$
I(u) \geq I\left(u_{\lambda}\right)+h^{\prime}\left(\lambda^{2}\right) \int_{\Omega}\left(\operatorname{det} \nabla u-\operatorname{det} \nabla u_{\lambda}\right) d x .
$$

Clearly, if the integral with prefactor $h^{\prime}\left(\lambda^{2}\right)$ vanishes, that is if

$$
\int_{\Omega}\left(\operatorname{det} \nabla u-\operatorname{det} \nabla u_{\lambda}\right) d x=0
$$

then $I(u) \geq I\left(u_{\lambda}\right)$ follows. This can be ensured, for example, by imposing further conditions on $u$ guaranteeing that

$$
\int_{\Omega} f(u(x)) \operatorname{det} \nabla u(x) d x=\int_{\mathbb{R}^{2}} f(y) \operatorname{deg}(\bar{u}, \partial \Omega, y) d y
$$

for any bounded continuous function $f$, where $\bar{u}$ represents the trace of $u$, here assumed to possess a continuous representative in order that the degree is well-defined. The idea behind this originates in Šverák's work [18], and was later refined by Müller et al. [12]. ${ }^{3}$ As Šverák remarks in [18], (2.3) clearly excludes cavitation by choosing $f$ with support in the created cavity. We note that (2.3) is a key ingredient in Šverák's proof of the existence of a representative for $u$ that is continuous outside a set of Hausdorff dimension $n-p$, where $p>n-1$ is the Sobolev exponent appearing in the class $\mathcal{A}_{p, q}^{+}$he works in: see [18] for further details of that rich theory. It turns out that the discrepancy between $\int_{\Omega} \operatorname{det} \nabla u d x$ and $\int_{\Omega} \operatorname{det} \nabla u_{\lambda} d x$ can be measured using Det $\nabla u$ and interpreted in terms of cavitation provided some additional conditions are imposed on $u$. To explain this we follow the approach in [14] and appeal to a result in [15] that is couched in terms of Müller and Spector's condition (INV). We now recall the definition of condition (INV), which is stated in terms of a general dimension $n$ and domain $\Omega$.

Definition 2.1 [15, Definition 3.2] The map $u: \Omega \rightarrow \mathbb{R}^{n}$ satisfies condition (INV) provided that for every $a \in \Omega$ there exists an $\mathcal{L}^{1}$-null set $N_{a}$ such that, for all $R \in(0$, dist $(a, \partial \Omega)) \backslash N_{a}$, $\left.u\right|_{S(a, R)}$ is continuous,

(i) $u(x) \in \operatorname{im}_{T}(u, B(a, R)) \cup u(S(a, R))$ for $\mathcal{L}^{n}$-a.e. $x \in \overline{B(a, R)}$, and

\footnotetext{
3 One could also produce (2.3) without reference to either of these papers. For example, (2.2) holds whenever $u$ is continuous, satisfies Lusin's $N$-property [i.e., $u$ maps sets of (Lebesgue) measure zero to sets of (Lebesgue) measure zero], and det $\nabla u$ belongs to $L^{1}(\Omega)$. See, for example, [7, Theorem 5.25].
} 
(ii) $u(x) \in \mathbb{R}^{n} \backslash \operatorname{im}_{T}(u, B(a, R))$ for $\mathcal{L}^{n}$-a.e. $x \in \Omega \backslash B(a, R)$.

The topological image of $B(a, R)$ under the mapping $u, \operatorname{im}_{T}(u, B(a, R))$, is defined below.

Lemma $2.2[15$, Lemma 8.1$]$ Let $u \in W^{1, q}\left(\Omega ; \mathbb{R}^{n}\right)$ with $q>n-1$. Suppose that det $\nabla u>$ 0 a.e. in $\Omega$ and that $u^{*}$, the precise representative ${ }^{4}$ of $u$, satisfies condition (INV). Then Det $\nabla u \geq 0$ and hence Det $\nabla u$ is a Radon measure. Furthermore,

$$
\text { Det } \nabla u=\operatorname{det} \nabla u \mathcal{L}^{n}+m
$$

where $m$ is singular with respect to Lebesgue measure and for $\mathcal{L}^{1}$-a.e. $R \in(0, \operatorname{dist}(a, \partial \Omega))$,

$$
(\operatorname{Det} \nabla u)(B(a, R))=\mathcal{L}^{n}\left(\operatorname{im}_{T}(u, B(a, R))\right) .
$$

Remark 2.3 Under the assumption that the perimeter of $\operatorname{im}_{T}(u, \Omega)$ is finite it can be shown that the singular part of Det $\nabla u$ is a sum of Dirac masses. Thus the left-hand side of (2.6) below is $-1 \times$ (volume of cavities created by the deformation $u$ ). See [15, Theorem 8.4] for more details.

Remark 2.4 Since $m$ is singular with respect to Lebesgue measure, and in view of Det $\nabla u \geq$ 0 , it is clear that $m \geq 0$.

Reverting to the two dimensional case $\Omega \subset \mathbb{R}^{2}$, the assumption that $u \in W^{1, q}(\Omega)$ for $q>1$ implies (by Sobolev embedding) that $\left.u\right|_{S(a, R)}$ is continuous for $\mathcal{L}^{1}$-a.e. $R \in(0$, dist $(a, \partial \Omega))$. Hence, for such $R$, the topological image

$$
\operatorname{im}_{T}(u, B(a, R))=\left\{y \in \mathbb{R}^{2} \backslash u(S(a, R)): \operatorname{deg}(u, S(a, R), y) \neq 0\right\}
$$

is well-defined. Following [14], we extend $u$ by setting it equal to $u_{\lambda}$ on $B(0, M) \backslash \bar{\Omega}$, where $M$ is chosen so that $\bar{\Omega} \subset B(0, M)$, and we assume that the extension satisfies condition (INV) on $B(0, M)$. It is then straightforward to check, using the definition of the distributional Jacobian, its representation through [15, Lemma 8.1] and (2.5), that

$$
-m(\bar{\Omega})=\int_{\Omega}\left(\operatorname{det} \nabla u-\operatorname{det} \nabla u_{\lambda}\right) d x
$$

Finally, by applying (2.6) to inequality (2.1), we obtain

$$
I(u) \geq I\left(u_{\lambda}\right)-h^{\prime}\left(\lambda^{2}\right) m(\bar{\Omega}) .
$$

It is clear that when $h^{\prime}\left(\lambda^{2}\right) \leq 0$ or $m(\bar{\Omega})=0$ we have $I(u) \geq I\left(u_{\lambda}\right)$. Summarising the above, we have the following:

Proposition 2.5 Suppose that $W(A)=|A|^{q}+h(\operatorname{det} A)$, where $h$ satisfies $(H 1)-(H 3)$, and where $q>1$. Let $B(0, M)$ contain $\bar{\Omega}$ and denote by $u^{e}$ the extension of $u$ to $B(0, M) \backslash \Omega$ defined by

$$
u^{e}(x):= \begin{cases}u(x) & \text { if } x \in \Omega, \\ u_{\lambda}(x) & \text { if } x \in B(0, M) \backslash \Omega .\end{cases}
$$

Assume that $u^{e}$ satisfies the hypotheses of [15, Lemma 8.1] in the case that $n=2$. Then if $\int_{\Omega} \operatorname{det} \nabla u d x=\int_{\Omega} \operatorname{det} \nabla u_{\lambda} d x$ or if $h^{\prime}\left(\lambda^{2}\right) \leq 0$, the inequality $I(u) \geq I\left(u_{\lambda}\right)$ holds.

${ }^{4}$ See $\left[15\right.$, p.13] for a definition of $u^{*}$. 
The rest of this section handles the case $h^{\prime}\left(\lambda^{2}\right)>0$ and $m(\bar{\Omega})>0$, where $m$ is given by (2.6), which is the situation not covered by Proposition 2.5. The following is a slightly improved version of a lemma by Zhang which, although stated here for general $n$, will only be needed in the case $n=2$.

Lemma 2.6 (Adaptation of [20, Lemma 2.15]) For $1<q<2, M>0$, and $A, B \in \mathbb{R}^{n \times n}$ with $0<|A| \leq M$,

$$
|A+B|^{q}-|A|^{q}-q|A|^{q-2} A \cdot B \geq \begin{cases}C_{1}(M, q)|B|^{2} & \text { if }|B| \leq M, \\ C_{2}(q)|B|^{q} & \text { if }|B| \geq M,\end{cases}
$$

The constants $C_{1}(M, q)$ and $C_{2}(q)$ are given by

$$
\begin{gathered}
C_{1}(M, q)=\frac{1}{2(2 M)^{2-q}}, \\
C_{2}(q)=\frac{1}{2\left(2^{2-q}\right)} .
\end{gathered}
$$

Proof The only part which requires proof is the constant $C_{2}(q)$ since it is larger than the original version $\tilde{C}_{2}(q):=\frac{1}{2\left(3^{2-q}\right)}$ given in [20, Lemma 2.14]. The constant $\tilde{C}_{2}(q)$ appears in [20, Eq. (2.23)] as a prefactor in the estimate

$$
\int_{0}^{1} \frac{(1-s)|B|^{2}}{|A+s B|^{2-q}} d s \geq \tilde{C}_{2}(q)|B|^{q}
$$

under the assumption that $|B| \geq M$. Now, in terms of $\tau:=|B| / M$,

$$
\begin{aligned}
\frac{(1-s)|B|^{2}}{|A+s B|^{2-q}} & \geq \frac{(1-s)|B|^{2}}{|M+s| B||^{2-q}} \\
& =\frac{(1-s) M^{q} \tau^{2}}{(1+s \tau)^{2-q}} \\
& \geq \frac{(1-s) \tau^{2-q}|B|^{q}}{(1+\tau)^{2-q} .}
\end{aligned}
$$

Since $\tau \geq 1$, the quantity $\frac{\tau^{2-q}}{(1+\tau)^{2-q}}$ is bounded below by $1 / 2^{2-q}$. Upon integration, the lower bound

$$
\int_{0}^{1} \frac{(1-s)|B|^{2}}{|A+s B|^{2-q}} d s \geq \frac{|B|^{q}}{2\left(2^{2-q}\right)}
$$

follows.

Let $u \in \mathcal{A}_{\lambda}$. Applying Lemma 2.6 to $A:=\lambda \mathbf{1}$ and $B:=\nabla u-\lambda \mathbf{1}$, we find that with $M:=|A|=\sqrt{2} \lambda$,

$$
|\nabla u|^{q} \geq|\lambda \mathbf{1}|^{q}+q|\lambda \mathbf{1}|^{q-2}(\nabla u-\lambda \mathbf{1}) \cdot \lambda \mathbf{1}+F_{M}(\nabla u-\lambda \mathbf{1})
$$

where the function $F_{M}: \mathbb{R}^{2 \times 2} \rightarrow \mathbb{R}$ is defined by

$$
F_{M}(B):= \begin{cases}C_{1}(M, q)|B|^{2} & \text { if }|B| \leq M \\ C_{2}(q)|B|^{q} & \text { if }|B| \geq M .\end{cases}
$$

Now

$$
|\nabla u-\lambda \mathbf{1}| \geq \operatorname{dist}(\nabla u, \lambda S O(2))
$$


and since, by polar factorization,

$$
\text { dist }(\nabla u, \lambda S O(2))=\left|\sqrt{\nabla u^{T} \nabla u}-\lambda \mathbf{1}\right|=\left|\left(\lambda_{1}(\nabla u), \lambda_{2}(\nabla u)\right)-(\lambda, \lambda)\right|,
$$

where $0<\lambda_{1}(\nabla u) \leq \lambda_{2}(\nabla u)$ are the singular values of $\nabla u$, we have

$$
|\nabla u-\lambda \mathbf{1}| \geq\left|\Lambda-\Lambda_{0}\right|
$$

Here, $\Lambda:=\left(\lambda_{1}, \lambda_{2}\right)$, where we leave out the dependence on $\nabla u$ for clarity, and $\Lambda_{0}:=(\lambda, \lambda)$. Next, define $f_{M}: \mathbb{R}^{+} \rightarrow \mathbb{R}^{+}$by

$$
f_{M}(t):=\min \left\{C_{1}(M, q) t^{2}, C_{2}(q) t^{q}\right\},
$$

where $C_{1}(M, q)$ and $C_{2}(q)$ are as in (2.8) and (2.9), respectively.

Remark 2.7 We note that $f_{M}$ is continuous on $\mathbb{R}^{+}$and $C_{1}(M, q) t^{2}=C_{2}(q) t^{q}$ if and only if $t=M$. Thus the growth of $f_{M}$ switches from quadratic on $[0, M]$ to $q$-growth on $[M,+\infty)$. We remark that the continuity is a consequence of the improved (i.e. increased) value for $C_{2}(q)$ provided in Lemma 2.6. More importantly, a larger value for $C_{2}(q)$ makes our estimate of the critical load more accurate: see (2.32), for example.

Then, by combining (2.11) and (2.12) with the definition of $F_{M}$, we obtain

$$
F_{M}(\nabla u-\lambda \mathbf{1}) \geq f_{M}\left(\left|\Lambda-\Lambda_{0}\right|\right) .
$$

Therefore, by (2.10),

$$
|\nabla u|^{q} \geq|\lambda \mathbf{1}|^{q}+q|\lambda \mathbf{1}|^{q-2}(\nabla u-\lambda \mathbf{1}) \cdot \lambda \mathbf{1}+f_{\sqrt{2} \lambda}\left(\left|\Lambda-\Lambda_{0}\right|\right) .
$$

Integrating this, applying the definition of the stored-energy function $W$, using

$$
\int_{\Omega}(\nabla u-\lambda \mathbf{1}) d x=0
$$

and recalling that det $\nabla u=\lambda_{1} \lambda_{2}$, gives

$$
I(u) \geq \int_{\Omega}\left(|\lambda \mathbf{1}|^{q}+f_{\sqrt{2} \lambda}\left(\left|\Lambda-\Lambda_{0}\right|\right)+h\left(\lambda_{1} \lambda_{2}\right)\right) d x .
$$

Then in view of the convexity of $h$ we get

$$
\begin{aligned}
I(u)-I\left(u_{\lambda}\right) & \geq \int_{\Omega} f_{\sqrt{2} \lambda}\left(\left|\Lambda-\Lambda_{0}\right|\right) d x+\int_{\Omega}\left(h\left(\lambda_{1} \lambda_{2}\right)-h\left(\lambda^{2}\right)\right) d x \\
& \geq \int_{\Omega} f_{\sqrt{2} \lambda}\left(\left|\Lambda-\Lambda_{0}\right|\right) d x+h^{\prime}\left(\lambda^{2}\right) \int_{\Omega}\left(\lambda_{1} \lambda_{2}-\lambda^{2}\right) d x .
\end{aligned}
$$

As has already been observed, we need only consider $h^{\prime}\left(\lambda^{2}\right)>0$, since Proposition 2.5 covers the case $h^{\prime}\left(\lambda^{2}\right) \leq 0$.

Note that

$$
f_{\sqrt{2} \lambda}\left(\left|\Lambda-\Lambda_{0}\right|\right)+h^{\prime}\left(\lambda^{2}\right)\left(\lambda_{1} \lambda_{2}-\lambda^{2}\right)=\mathcal{G}_{1}^{\lambda}(\Lambda)+\mathcal{G}_{2}^{\lambda}(\Lambda),
$$

where

$$
\mathcal{G}_{1}^{\lambda}(\Lambda):=f_{\sqrt{2} \lambda}\left(\left|\Lambda-\Lambda_{0}\right|\right)+h^{\prime}\left(\lambda^{2}\right)\left(\lambda_{1}-\lambda\right)\left(\lambda_{2}-\lambda\right)
$$

and

$$
\mathcal{G}_{2}^{\lambda}(\Lambda):=\lambda h^{\prime}\left(\lambda^{2}\right)\left(\lambda_{1}+\lambda_{2}-2 \lambda\right),
$$


so that we have

$$
I(u)-I\left(u_{\lambda}\right) \geq \int_{\Omega} \mathcal{G}_{1}^{\lambda}(\Lambda) d x+\int_{\Omega} \mathcal{G}_{2}^{\lambda}(\Lambda) d x .
$$

The rest of this section is devoted to finding conditions on $\lambda$ which ensure that

$$
\int_{\Omega} \mathcal{G}_{i}^{\lambda}(\Lambda) d x \geq 0 \text { for } i=1,2 .
$$

The following result, in which inequality (2.16) is part of [2, Lemma 5.3], allows us to deal with the term involving $\mathcal{G}_{2}^{\lambda}$. We give a short elementary proof here to keep the paper selfcontained; we also give a refined version of the estimate (2.16) which provides an 'excess term' [an estimate of the difference between the two sides of the inequality (2.16)]: see (2.17) below.

Lemma 2.8 Let $u \in W^{1,1}\left(\Omega, \mathbb{R}^{2}\right)$ satisfy $u=u_{\lambda}$ on $\partial \Omega$ and suppose that $\operatorname{det} \nabla u>0$ a.e. in $\Omega$. Then

$$
\int_{\Omega}\left(\lambda_{1}+\lambda_{2}\right) d x \geq 2 \lambda \mathcal{L}^{2}(\Omega)
$$

where $0<\lambda_{1} \leq \lambda_{2}$ denote the singular values of $\nabla u$. Moreover,

$$
\int_{\Omega}\left(\lambda_{1}+\lambda_{2}-2 \lambda\right) d x \geq \int_{\Omega} \psi(u, \lambda) d x
$$

where

$$
\psi(u, \lambda):=\frac{2 \lambda^{2}(\operatorname{curl} u)^{2}}{\left((\operatorname{curl} u)^{2}+\max \left\{4 \lambda^{2},(\operatorname{div} u)^{2}\right\}\right)^{\frac{3}{2}}} .
$$

Proof We first give a direct proof of (2.16).

The singular value decomposition theorem (see e.g., [6, Theorem 13.3]) yields

$$
\nabla u=R D\left(\lambda_{1}, \lambda_{2}\right) Q
$$

where $R, Q \in O(2)$ and

$$
D\left(\lambda_{1}, \lambda_{2}\right):=\left(\begin{array}{cc}
\lambda_{1} & 0 \\
0 & \lambda_{2}
\end{array}\right)
$$

Hence

$$
\operatorname{tr} \nabla u=\operatorname{tr}\left(Q R D\left(\lambda_{1}, \lambda_{2}\right)\right) .
$$

Since $Q R \in O(2)$, it must be of the form

$$
Q R=\left(\begin{array}{ll}
\cos \sigma & \pm \sin \sigma \\
\sin \sigma & \mp \cos \sigma
\end{array}\right)
$$

therefore

$$
\operatorname{tr} \nabla u=\cos \sigma\left(\lambda_{1} \mp \lambda_{2}\right)
$$

It can now be checked that

$$
\operatorname{tr} \nabla u \leq \lambda_{1}+\lambda_{2} .
$$


Then integrating the latter expression over $\Omega$ and using the fact that the weak derivative satisfies

$$
\int_{\Omega} \operatorname{tr} \nabla u d x=\int_{\Omega} \operatorname{tr} \nabla u_{\lambda} d x=2 \lambda \mathcal{L}^{2}(\Omega)
$$

yields (2.16).

To prove (2.17), let $\xi \in \mathbb{R}^{2 \times 2}$, denote by $\lambda_{1}(\xi), \lambda_{2}(\xi)$ the singular values of $\xi$ and define the function $\varphi: \mathbb{R}^{2 \times 2} \rightarrow[0,+\infty)$ by

$$
\varphi(\xi):=\lambda_{1}(\xi)+\lambda_{2}(\xi)
$$

Notice that

$$
\varphi(\xi)=\sqrt{|\xi|^{2}+2 \operatorname{det} \xi} .
$$

Then by applying the standard identity

$$
g(1)=g(0)+g^{\prime}(0)+\int_{0}^{1}(1-s) g^{\prime \prime}(s) d s
$$

to the function $g(s):=\varphi((1-s) \lambda \mathbf{1}+s \xi)$ defined for $s \in[0,1]$, we obtain

$$
\begin{aligned}
\varphi(\xi)= & \varphi(\lambda \mathbf{1})+\operatorname{tr}(\xi-\lambda \mathbf{1}) \\
& +\int_{0}^{1}(1-s) \frac{\varphi^{2}(\omega(s)) \varphi^{2}(\xi-\lambda \mathbf{1})-((\omega(s)+\operatorname{cof} \omega(s)) \cdot(\xi-\lambda \mathbf{1}))^{2}}{\varphi^{3}(\omega(s))} d s
\end{aligned}
$$

where

$$
\omega(s):=(1-s) \lambda \mathbf{1}+s \xi \text { for } 0 \leq s \leq 1 .
$$

For later use we note that the term

$$
X(\omega(s), \xi-\lambda \mathbf{1}):=\frac{\varphi^{2}(\omega(s)) \varphi^{2}(\xi-\lambda \mathbf{1})-((\omega(s)+\operatorname{cof} \omega(s)) \cdot(\xi-\lambda \mathbf{1}))^{2}}{\varphi^{3}(\omega(s))}
$$

can be rewritten as

$$
X(\omega(s), \xi-\lambda \mathbf{1})=\frac{(\operatorname{atr}(\omega(s)) \operatorname{tr}(\xi-\lambda \mathbf{1})-\operatorname{atr}(\xi-\lambda \mathbf{1}) \operatorname{tr}(\omega(\mathrm{s})))^{2}}{\varphi^{3}(\omega(s))} .
$$

Here, $\operatorname{atr}(\eta)$ denotes the antitrace of any $\eta \in \mathbb{R}^{2 \times 2}$ and is defined by $\operatorname{atr}(\eta):=\eta_{12}-\eta_{21}$. Note that, thanks to $(2.21), X(\cdot, \cdot) \geq 0$ for all $\xi$ and $s \in[0,1]$, so that by letting $\xi=\nabla u$ in (2.20) we obtain an alternative proof of (2.16).

Then (2.17) follows by calculating the terms in (2.21). Letting $\xi=\nabla u$ again, we have $\omega(s)=\lambda \mathbf{1}+s(\nabla u-\lambda \mathbf{1})$, and

$$
\begin{aligned}
\operatorname{atr}(\nabla u-\lambda \mathbf{1}) & =\operatorname{curl} u \\
\operatorname{tr}(\nabla u-\lambda \mathbf{1}) & =\operatorname{div} u-2 \lambda \\
\operatorname{atr}(\omega(s)) & =s \operatorname{curl} u \\
\operatorname{tr}(\omega(s)) & =s \operatorname{div} u+(1-s) 2 \lambda .
\end{aligned}
$$

This gives

$$
X(\omega(s), \xi-\lambda \mathbf{1})=\frac{4 \lambda^{2}(\operatorname{curl} u)^{2}}{\varphi^{3}(\lambda \mathbf{1}+s(\nabla u-\lambda \mathbf{1}))}
$$


Now

$$
\varphi^{2}(\eta)=(\operatorname{atr}(\eta))^{2}+(\operatorname{tr}(\eta))^{2}
$$

so we have

$$
\varphi^{2}(\lambda \mathbf{1}+s(\nabla u-\lambda \mathbf{1}))=s^{2}(\operatorname{curl} u)^{2}+(s \operatorname{div} u+2(1-s) \lambda)^{2} .
$$

Since the function

$$
p: s \mapsto(s \operatorname{div} u+2(1-s) \lambda)^{2}
$$

is convex, its maximum on the interval $[0,1]$ must be $\max \{p(0), p(1)\}$. Hence

$$
\varphi^{2}(\lambda \mathbf{1}+s(\nabla u-\lambda \mathbf{1})) \leq(\operatorname{curl} u)^{2}+\max \left\{4 \lambda^{2},(\operatorname{div} u)^{2}\right\}
$$

uniformly in $s$. Therefore (2.22) gives

$$
X(\omega(s), \xi-\lambda \mathbf{1}) \geq \frac{4 \lambda^{2}(\operatorname{curl} u)^{2}}{\left((\operatorname{curl} u)^{2}+\max \left\{4 \lambda^{2},(\operatorname{div} u)^{2}\right\}\right)^{\frac{3}{2}}} .
$$

Inserting this into (2.20), recalling that

$$
\lambda_{1}+\lambda_{2}-2 \lambda=\varphi(\nabla u)-\varphi(\lambda \mathbf{1}),
$$

and carrying out what becomes a trivial integration yields (2.17).

We now return to the estimate of $\mathcal{G}_{2}^{\lambda}$. Indeed, since we are working under the assumption $\lambda h^{\prime}\left(\lambda^{2}\right)>0$ for every $\lambda>0$, applying Lemma 2.8 gives

$$
\int_{\Omega} \mathcal{G}_{2}^{\lambda}(\Lambda) d x \geq 0
$$

as desired.

To deal with the term involving $\mathcal{G}_{1}^{\lambda}$ we find an explicit condition on $\lambda$ which ensures that $\mathcal{G}_{1}^{\lambda}(\Lambda) \geq 0$ holds pointwise for $\Lambda \in \mathbb{R}^{++}$where

$$
\mathbb{R}^{++}:=\left\{x \in \mathbb{R}^{2}: x_{1}, x_{2}>0\right\}
$$

Lemma 2.9 The function

$$
\mathcal{G}_{1}^{\lambda}(\Lambda)=f_{\sqrt{2} \lambda}\left(\left|\Lambda-\Lambda_{0}\right|\right)+h^{\prime}\left(\lambda^{2}\right)\left(\lambda_{1}-\lambda\right)\left(\lambda_{2}-\lambda\right)
$$

is pointwise nonnegative on $\mathbb{R}^{++}$provided

$$
C_{1}(\sqrt{2} \lambda, q) \geq h^{\prime}\left(\lambda^{2}\right) / 2
$$

and

$$
\frac{C_{2}(q)}{h^{\prime}\left(\lambda^{2}\right) \lambda^{2-q}} \geq(q-1)^{(q-1) / 2} q^{-q / 2} .
$$

Moreover, inequality (2.25) implies (2.24). 
Proof We divide the proof into two parts, the first of which is devoted to proving the sufficiency of (2.24) and (2.25).

Part 1 To shorten notation set $Y:=h^{\prime}\left(\lambda^{2}\right)$. Let $\Lambda-\Lambda_{0}=\left(\lambda_{1}-\lambda, \lambda_{2}-\lambda\right)=$ $(\rho \cos \mu, \rho \sin \mu)$ and let $C_{1}:=C_{1}(\sqrt{2} \lambda, q)$ and $C_{2}:=C_{2}(q)$, as defined in (2.8) and (2.9) respectively. Let $G(\rho, \mu):=\mathcal{G}_{1}^{\lambda}(\Lambda)$ and note that (using (2.12) with $M=\sqrt{2} \lambda$ )

$$
G(\rho, \mu)= \begin{cases}C_{1} \rho^{2}+Y \rho^{2} \sin \mu \cos \mu & \text { if } \rho \leq \sqrt{2} \lambda \\ C_{2} \rho^{q}+Y \rho^{2} \sin \mu \cos \mu & \text { if } \rho \geq \sqrt{2} \lambda .\end{cases}
$$

Firstly, if $\rho \leq \sqrt{2} \lambda$ then $G(\rho, \mu) \geq 0$ if and only if $C_{1}+Y \sin \mu \cos \mu \geq 0$ for all $\mu$. Whence $C_{1}-Y / 2 \geq 0$, which is (2.24). We henceforth suppose that (2.24) holds.

Inequality (2.25) essentially prevents $G(\rho, \mu)$ from vanishing at any point in $\mathbb{R}^{++}$outside the set $B\left(\Lambda_{0}, \sqrt{2} \lambda\right) \cap \mathbb{R}^{++}$. By symmetry, we need only consider $\mu \in[-\pi / 4, \pi / 4]$, and since $G(\rho, \mu) \geq 0$ if $0 \leq \mu \leq \pi / 4$, we can restrict attention to $-\pi / 4<\mu \leq 0$. Moreover, since $G(\rho, 0)$ is obviously nonegative, we can also exclude $\mu=0$. Now, in view of (2.24), the only way $G(\rho, \mu)$ can vanish is if $\rho \geq \sqrt{2} \lambda$. In the region $\rho \geq \sqrt{2} \lambda,-\pi / 4<\mu<0$

$$
G(\rho, \mu)=C_{2} \rho^{q}-Y|\sin \mu \cos \mu| \rho^{2},
$$

and since $1<q<2$, it must be that $G(\rho, \mu)<0$ for sufficiently large $\rho$ and each fixed $\mu$. Also, since $G(\rho, \mu)$ is continuous and since, by $(2.24), G(\sqrt{2} \lambda, \mu) \geq 0$, it follows that

$$
\bar{\rho}(\mu):=\inf \left\{\rho \geq \sqrt{2} \lambda: C_{2} \rho^{q}-Y|\sin \mu \cos \mu| \rho^{2}=0\right\}
$$

is well-defined. Thus $\bar{\rho}(\mu)$ satisfies

$$
C_{2} \bar{\rho}(\mu)^{q}-Y|\sin \mu \cos \mu| \bar{\rho}(\mu)^{2}=0 .
$$

Now, if the point $(\bar{\rho}(\mu) \cos \mu+\lambda, \bar{\rho}(\mu) \sin \mu+\lambda)$ lies in the interior of $\mathbb{R}^{++}$then, by making $\rho$ slightly larger, we ensure $G(\rho, \mu)<0$. Since $-\pi / 4<\mu<0$, the inclusion

$$
(\bar{\rho}(\mu) \cos \mu+\lambda, \bar{\rho}(\mu) \sin \mu+\lambda) \in \mathbb{R}^{++}
$$

is prevented when and only when

$$
\bar{\rho}(\mu) \geq \rho^{*}(\mu),
$$

where $\rho^{*}(\mu)$ satisfies $\rho^{*}(\mu) \sin \mu+\lambda=0$ and $-\pi / 4<\mu<0$.

Using (2.27) and the definition of $\rho^{*}$, inequality (2.28) is equivalent to

$$
\frac{C_{2}}{Y \lambda^{2-q}} \geq \underbrace{\cos \mu|\sin \mu|^{q-1}}_{=: e(\mu)},
$$

where $-\pi / 4<\mu<0$. It can be checked that

$$
\max _{(-\pi / 4,0)} e=(q-1)^{(q-1) / 2} q^{-q / 2},
$$

the maximum occurring at $\mu$ such that $\cos ^{2} \mu=1 / q$. Inequality (2.25) now follows.

Part 2 We prove that (2.25) implies (2.24). First note that dividing both sides of (2.25) by $2^{(2-q) / 2}$ gives

$$
\frac{C_{1}(\sqrt{2} \lambda, q)}{Y} \geq \underbrace{\left(\frac{(q-1)^{q-1} q^{-q}}{2^{2-q}}\right)^{1 / 2}}_{=: y(q)} .
$$


Let $\gamma(q)=2 \ln y(q)$ and calculate $\gamma^{\prime}(q)=\ln \left(2\left(1-\frac{1}{q}\right)\right)$. Now $1<q<2$, so $2\left(1-\frac{1}{q}\right) \in(0,1)$, and hence $\gamma^{\prime}(q)<0$ on $(1,2)$. It follows that $y$ is a decreasing function of $q$ on $(1,2)$, and since $y(q) \rightarrow \frac{1}{2}$ as $q \rightarrow 2-$, the right-hand side of (2.31) is bounded below by $\frac{1}{2}$. Hence (2.24) holds.

We now draw the preceding discussions together in the following result, whose statement, in contrast to that of Proposition 2.5, does not rely on the imposition of condition (INV).

Theorem 2.10 Let the stored energy function $W: \mathbb{R}^{2 \times 2} \rightarrow[0,+\infty]$ be given by

$$
W(A):=|A|^{q}+h(\operatorname{det} A),
$$

where $1<q<2$ and $h: \mathbb{R} \rightarrow[0,+\infty]$ satisfies $(H 1)-(H 3)$. Let $\lambda>0$ be such that

$$
\frac{1}{2^{3-q} h^{\prime}\left(\lambda^{2}\right) \lambda^{2-q}} \geq(q-1)^{(q-1) / 2} q^{-q / 2} .
$$

Then any $u \in \mathcal{A}_{\lambda}$ satisfies $I(u) \geq I\left(u_{\lambda}\right)$.

\subsection{Error estimates}

In this section we are interested in understanding the properties of those $u \in \mathcal{A}_{\lambda}$ such that $I(u)-I\left(u_{\lambda}\right)$ is small and positive. Hence we focus on the case $h^{\prime}\left(\lambda^{2}\right)>0$ to which the results of the previous section apply. Accordingly, we impose the hypotheses of Theorem 2.10 and strengthen inequality (2.32) to read

$$
\frac{1}{2^{3-q} h^{\prime}\left(\lambda^{2}\right) \lambda^{2-q}}>(q-1)^{(q-1) / 2} q^{-q / 2} .
$$

The main result of this subsection is the following.

Theorem 2.11 Assume that (2.33) holds. Then there is a constant $c=c(\Omega, \lambda, q)>0$ such that for every $u \in \mathcal{A}_{\lambda}$

$$
\int_{\Omega} \min \left\{|\nabla u-\lambda \mathbf{1}|^{2},|\nabla u-\lambda \mathbf{1}|^{q}\right\} d x \leq c \delta(u),
$$

where $\delta(u):=I(u)-I\left(u_{\lambda}\right)$. Moreover,

$$
\lambda h^{\prime}\left(\lambda^{2}\right) \int_{\Omega} \frac{2 \lambda^{2}(\operatorname{curl} u)^{2}}{\left((\operatorname{curl} u)^{2}+\max \left\{4 \lambda^{2},(\operatorname{div} u)^{2}\right\}\right)^{\frac{3}{2}}} d x \leq \delta(u) .
$$

The proof of Theorem 2.11 is given in stages below. In view of

$$
\int_{\Omega} \mathcal{G}_{1}^{\lambda}(\Lambda) d x+\int_{\Omega} \mathcal{G}_{2}^{\lambda}(\Lambda) d x \leq \delta(u),
$$

the idea is that if $\delta(u)$ is small then the same must be true of the two (necessarily nonnegative) terms in the left-hand side of (2.36). The first inequality, (2.34), follows from a smallness assumption on $\int_{\Omega} \mathcal{G}_{1}^{\lambda}(\Lambda) d x$ : see Proposition 2.14 below, while inequality (2.35) is a consequence of small $\int_{\Omega} \mathcal{G}_{2}^{\lambda}(\Lambda) d x$ and follows in a straightforward way from (2.17).

We remark that an inequality like (2.35) is not available in the three dimensional case, or at least we could not derive it. The chief difficulty is the lack of an explicit expression for $\lambda_{1}(\xi)+\lambda_{2}(\xi)+\lambda_{3}(\xi)$ for $\xi \in \mathbb{R}^{3 \times 3}$ : cf. (2.18) and (2.19). 
We now turn to inequality (2.34). To this end we introduce the function $g:[0,+\infty) \rightarrow$ $[0,+\infty)$ defined by

$$
g(t):= \begin{cases}\frac{t^{2}}{2} & \text { if } 0 \leq t \leq 1, \\ \frac{t^{q}}{q}+\frac{1}{2}-\frac{1}{q} & \text { if } t \geq 1\end{cases}
$$

For later use we notice that $g$ is convex.

Lemma 2.12 Let (2.33) hold. Then there is a constant $c_{0}=c_{0}(\lambda, q)>0$ such that

$$
\mathcal{G}_{1}^{\lambda}(\Lambda) \geq c_{0} g\left(\left|\Lambda-\Lambda_{0}\right|\right) \text { on } \mathbb{R}^{++}
$$

where $g$ is as in (2.37).

Proof It is clear from the last part of the proof of Lemma 2.9 that inequality (2.33) implies that (2.24) holds with strict inequality. Thus

$$
\mathcal{G}_{1}^{\lambda}(\Lambda) \geq c\left|\Lambda-\Lambda_{0}\right|^{2} \quad \text { if }\left|\Lambda-\Lambda_{0}\right| \leq \sqrt{2} \lambda
$$

for some constant $c>0$.

Reusing the notation $\Lambda-\Lambda_{0}=\rho(\cos \mu, \sin \mu)$ and $G(\rho, \mu):=\mathcal{G}_{1}^{\lambda}(\Lambda)$, the case $\rho \geq \sqrt{2} \lambda$ can be handled as follows. Let $\epsilon>0$ and write

$$
\begin{aligned}
G(\rho, \mu) & =C_{2} \rho^{q}-Y|\sin \mu \cos \mu| \rho^{2} \\
& =\left(C_{2}-\epsilon\right) \rho^{q}-Y|\sin \mu \cos \mu| \rho^{2}+\epsilon \rho^{q},
\end{aligned}
$$

where $Y:=h^{\prime}\left(\lambda^{2}\right)$. By applying the reasoning in the proof of Lemma 2.9 to the function

$$
\tilde{G}(\rho, \mu):=\left(C_{2}-\epsilon\right) \rho^{q}-Y|\sin \mu \cos \mu| \rho^{2},
$$

we see that $\tilde{G}(\rho, \mu) \geq 0$ provided

$$
\frac{C_{2}-\epsilon}{Y \lambda^{2-q}} \geq(q-1)^{(q-1) / 2} q^{-q / 2} .
$$

Inequality (2.33) clearly implies that $C_{2}$ exceeds the right-hand side of (2.40) by a fixed amount; thus, if $\epsilon>0$ is sufficiently small, inequality (2.40) holds. Hence

$$
\mathcal{G}_{1}^{\lambda}(\Lambda) \geq \epsilon\left|\Lambda-\Lambda_{0}\right|^{q} \quad \text { if }\left|\Lambda-\Lambda_{0}\right| \geq \sqrt{2} \lambda .
$$

Inequalities (2.39) and (2.41) are easily combined to give (2.38).

We will see that inequality (2.34) is a consequence of the $L^{2}+L^{q}$ rigidity estimate [5, Theorem 1.1], or of [13, Proposition 2.3]. We recall here the following variant (see [1, Lemma 3.1]) which is suitable for our purposes.

Lemma 2.13 Let $U \subset \mathbb{R}^{n}$ be a bounded domain with Lipschitz boundary. Let $\lambda>0$ and $g$ be as in (2.37). There exists a constant $c=c(U, \lambda, q)>0$ with the following property: for every $v \in W^{1, q}\left(U ; \mathbb{R}^{n}\right)$ there is a constant rotation $R \in S O(n)$ satisfying

$$
\int_{U} g(|\nabla v-\lambda R|) d x \leq c \int_{U} g(\operatorname{dist}(\nabla v, \lambda S O(n))) d x .
$$


Proof Once we observe that, thanks to [8, Theorem 3.1] we can find $c=c(U)>0$ such that for every $w \in W^{1,2}\left(U ; \mathbb{R}^{n}\right)$ there is a constant rotation $R \in S O(n)$ satisfying

$$
\int_{U}|\nabla w-\lambda R|^{2} d x \leq c \int_{U} \operatorname{dist}^{2}(\nabla w, \lambda S O(n)) d x
$$

the proof then closely follows that of [1, Lemma 3.1].

Proposition 2.14 There is a constant $c=c(\Omega, \lambda, q)>0$ such that

$$
\int_{\Omega} \min \left\{|\nabla u-\lambda \mathbf{1}|^{2},|\nabla u-\lambda \mathbf{1}|^{q}\right\} d x \leq c \delta(u) .
$$

Proof Throughout this proof $c$ denotes a generic strictly positive constant possibly depending on $\Omega, \lambda$, and $q$. By (2.23) and (2.36) we have

$$
\int_{\Omega} \mathcal{G}_{1}^{\lambda}(\Lambda) d x \leq \delta(u)
$$

Hence on recalling that

$$
\left|\Lambda-\Lambda_{0}\right|=\operatorname{dist}(\nabla u, \lambda S O(2)),
$$

and by appealing to Lemma 2.12 , we get

$$
c_{0} \int_{\Omega} g(\operatorname{dist}(\nabla u, \lambda S O(2))) d x \leq \delta(u) .
$$

Then Lemma 2.13 provides us with $c>0$ and $R \in S O(2)$ such that

$$
\int_{\Omega} g(|\nabla u-\lambda R|) d x \leq c \delta(u) .
$$

We claim that

$$
|\mathbf{1}-R|^{2} \leq c \delta(u)
$$

By virtue of the convexity of $g$, combining Jensen's inequality with (2.43) gives

$$
g\left(\frac{1}{\mathcal{L}^{2}(\Omega)} \int_{\Omega}|\nabla u-\lambda R| d x\right) \leq c \delta(u) .
$$

Set $\tilde{u}:=u / \lambda$ and $\tilde{z}:=\frac{1}{\mathcal{L}^{2}(\Omega)} \int_{\Omega}(\tilde{u}-R x) d x$. Then by Poincaré's inequality together with the continuity of the trace operator we obtain

$$
\int_{\partial \Omega}|\tilde{u}-R x-\tilde{z}| d \mathcal{H}^{1} \leq c \int_{\Omega}|\nabla \tilde{u}-R| d x
$$

and hence, since $\tilde{u}=x$ on $\partial \Omega$, we deduce that

$$
\int_{\partial \Omega}|(\mathbf{1}-R) x-\tilde{z}| d \mathcal{H}^{1} \leq c \int_{\Omega}|\nabla \tilde{u}-R| d x .
$$

Arguing as in the proof of [1, Lemma 3.3], we apply [1, Lemma 3.2] to deduce that there exists a universal constant $\sigma>0$ such that

$$
|\mathbf{1}-R| \leq \sigma \min _{z \in \mathbb{R}^{2}} \int_{\partial \Omega}|(\mathbf{1}-R) x-z| d \mathcal{H}^{1} .
$$


Combining (2.46) and (2.47) gives

$$
\begin{aligned}
|\mathbf{1}-R| & \leq c \int_{\Omega}|\nabla \tilde{u}-R| d x \\
& =\frac{c}{\lambda} \int_{\Omega}|\nabla u-\lambda R| d x,
\end{aligned}
$$

and therefore

$$
|\mathbf{1}-R|^{2} \leq c\left(\frac{1}{\mathcal{L}^{2}(\Omega)} \int_{\Omega}|\nabla u-\lambda R| d x\right)^{2} .
$$

Then to prove (2.44) we need to distinguish two cases.

(i) $\int_{\Omega}|\nabla u-\lambda R| d x \leq \mathcal{L}^{2}(\Omega)$. By definition $g(t)=t^{2} / 2$ for $t \leq 1$, so that (2.45) and $(2.48)$ immediately yield

$$
|\mathbf{1}-R|^{2} \leq \operatorname{cg}\left(\frac{1}{\mathcal{L}^{2}(\Omega)} \int_{\Omega}|\nabla u-\lambda R| d x\right) \leq c \delta(u) .
$$

(ii) $\int_{\Omega}|\nabla u-\lambda R| d x>\mathcal{L}^{2}(\Omega)$.

When $t>1$ we have $g(t)>1 / 2$, then

$$
\begin{aligned}
|\mathbf{1}-R|^{2} & \leq 2\left(|\mathbf{1}|^{2}+|R|^{2}\right) \\
& <\operatorname{cg}\left(\frac{1}{\mathcal{L}^{2}(\Omega)} \int_{\Omega}|\nabla u-\lambda R| d x\right) \\
& \leq \operatorname{cc} \delta(u),
\end{aligned}
$$

hence the claim is proved.

We now notice that the convexity of $g$ together with its definition entails

$$
g(s+t) \leq c\left(g(s)+t^{2}\right) \text { for every } s, t \geq 0
$$

and for some $c>0$. Indeed we have

$$
\begin{aligned}
g(s+t) & \leq 2^{q} g\left(\frac{s+t}{2}\right) \\
& \leq 2^{q-1}(g(s)+g(t)) \\
& \leq 2^{q-1}\left(g(s)+\frac{t^{2}}{q}\right) .
\end{aligned}
$$

Then choosing $R$ as in (2.43) and combining the latter with (2.44) implies

$$
\begin{aligned}
\int_{\Omega} g(|\nabla u-\lambda \mathbf{1}|) d x & =\int_{\Omega} g(|\nabla u-\lambda R+\lambda R-\lambda \mathbf{1}|) d x \\
& \leq c\left(\int_{\Omega} g(|\nabla u-\lambda R|) d x+\lambda^{2}|\mathbf{1}-R|^{2}\right) \\
& \leq c \delta(u) .
\end{aligned}
$$

Finally, since we can find $c>0$ such that

$$
\min \left\{t^{2}, t^{q}\right\} \leq \operatorname{cg}(t) \text { for every } t \geq 0,
$$


we obtain

$$
\int_{\Omega} \min \left\{|\nabla u-\lambda \mathbf{1}|^{2},|\nabla u-\lambda \mathbf{1}|^{q}\right\} d x \leq c \delta(u),
$$

which is the thesis.

Remark 2.15 Using (2.27) and the definition of $g$ we obtain

$$
\int_{|\nabla u-\lambda \mathbf{1}| \leq 1}|\nabla u-\lambda \mathbf{1}|^{2} d x \leq c \int_{\Omega} g(|\nabla u-\lambda \mathbf{1}|) d x \leq c \delta(u) .
$$

Then recalling that $q<2$, Hölder's inequality combined with (2.50) yields

$$
\int_{|\nabla u-\lambda \mathbf{1}| \leq 1}|\nabla u-\lambda \mathbf{1}|^{q} d x \leq \mathcal{L}^{2}(\Omega)^{1-\frac{q}{2}}\left(\int_{|\nabla u-\lambda \mathbf{1}| \leq 1}|\nabla u-\lambda \mathbf{1}|^{2} d x\right)^{\frac{q}{2}} \leq c \delta(u)^{\frac{q}{2}} .
$$

On the other hand we clearly have

$$
\int_{|\nabla u-\lambda \mathbf{1}|>1}|\nabla u-\lambda \mathbf{1}|^{q} d x \leq c \int_{\Omega} g(|\nabla u-\lambda \mathbf{1}|) d x \leq c \delta(u) .
$$

Therefore (2.51) and (2.52) together give

$$
\int_{\Omega}|\nabla u-\lambda \mathbf{1}|^{q} d x \leq c\left(\delta(u)^{\frac{q}{2}}+\delta(u)\right),
$$

which on applying Poincaré's inequality finally implies

$$
\left\|u-u_{\lambda}\right\|_{W^{1, q}\left(\Omega ; \mathbb{R}^{2}\right)}^{q} \leq c\left(\delta(u)^{\frac{q}{2}}+\delta(u)\right) .
$$

If $\lambda$ satisfies (2.33) then from (2.53) we can conclude that $u_{\lambda}$ is the unique global minimiser of $I$ among all maps $u$ in $\mathcal{A}_{\lambda}$ and, moreover, that $u_{\lambda}$ lies in a potential well.

\section{The three dimensional case}

In this section we seek conditions analogous to those obtained in the two dimensional case ensuring that $u_{\lambda}$ is the unique global minimizer of an appropriately defined stored-energy function. For simplicity we focus on the following $W: \mathbb{R}^{3 \times 3} \rightarrow[0,+\infty]$ given by

$$
W(A):=|A|^{q}+\gamma|A|^{2}+Z(\operatorname{cof} A)+h(\operatorname{det} A),
$$

where $2<q<3, \gamma>0$ is a fixed constant, $Z: \mathbb{R}^{3 \times 3} \rightarrow[0,+\infty)$ is convex and $C^{1}$, and $h$ has properties $(\mathrm{H} 1)-(\mathrm{H} 3)$.

Applying [14, Lemma A.1] to $A \mapsto|A|^{q}$ gives

$$
|\nabla u|^{q} \geq|\lambda \mathbf{1}|^{q}+q|\lambda \mathbf{1}|^{q-2} \lambda \mathbf{1} \cdot(\nabla u-\lambda \mathbf{1})+\kappa|\nabla u-\lambda \mathbf{1}|^{q},
$$

where

$$
2^{2-q} \leq \kappa \leq q 2^{1-q}
$$

Moreover, we clearly have

$$
\gamma|\nabla u|^{2} \geq \gamma|\lambda \mathbf{1}|^{2}+2 \gamma \lambda \mathbf{1} \cdot(\nabla u-\lambda \mathbf{1})+\gamma|\nabla u-\lambda \mathbf{1}|^{2} .
$$


Therefore, by gathering (3.2) and (3.4) and appealing to the convexity of $Z$ and $h$, we obtain

$$
\begin{aligned}
W(\nabla u) \geq & W\left(\nabla u_{\lambda}\right)+q|\lambda \mathbf{1}|^{q-2} \lambda \mathbf{1} \cdot(\nabla u-\lambda \mathbf{1})+\kappa|\nabla u-\lambda \mathbf{1}|^{q} \\
& +2 \gamma \lambda \mathbf{1} \cdot(\nabla u-\lambda \mathbf{1})+\gamma|\nabla u-\lambda \mathbf{1}|^{2} \\
& +D_{A} Z(\operatorname{cof} \lambda \mathbf{1}) \cdot(\operatorname{cof} \nabla u-\operatorname{cof} \lambda \mathbf{1}) \\
& +h^{\prime}\left(\lambda^{3}\right)\left(\operatorname{det} \nabla u-\lambda^{3}\right)
\end{aligned}
$$

for any $u \in \mathcal{A}_{\lambda}$, where $\mathcal{A}_{\lambda}$ is the class of admissible maps given by (1.4) with $n=3$. Integrating (3.5) and using the facts that both $\nabla u$ and cof $\nabla u$ are null Lagrangians in $W^{1, q}\left(\Omega, \mathbb{R}^{3}\right)$ for $q \geq 2$, we obtain

$$
I(u)-I\left(u_{\lambda}\right) \geq \int_{\Omega}\left(\kappa|\nabla u-\lambda \mathbf{1}|^{q}+\gamma|\nabla u-\lambda \mathbf{1}|^{2}+h^{\prime}\left(\lambda^{3}\right)\left(\operatorname{det} \nabla u-\lambda^{3}\right)\right) d x
$$

By analogy with Proposition 2.5 we can deal with the case $h^{\prime}\left(\lambda^{3}\right) \leq 0$ by imposing condition (INV) on a suitably defined extension of $u$, as follows.

Proposition 3.1 Suppose that $W: \mathbb{R}^{3 \times 3} \rightarrow[0,+\infty]$ is given by

$$
W(A):=|A|^{q}+\gamma|A|^{2}+Z(\operatorname{cof} A)+h(\operatorname{det} A)
$$

where $2<q<3, \gamma>0$ is a fixed constant, $Z: \mathbb{R}^{3 \times 3} \rightarrow[0,+\infty)$ is convex and $C^{1}$, and $h$ has properties $(H 1)-(H 3)$. Let $B(0, M)$ contain $\bar{\Omega}$ and denote by $u^{\mathrm{e}}$ the extension of $u$ to $B(0, M) \backslash \Omega$ defined by

$$
u^{\mathrm{e}}(x):= \begin{cases}u(x) & \text { if } x \in \Omega, \\ u_{\lambda}(x) & \text { if } x \in B(0, M) \backslash \Omega .\end{cases}
$$

Assume that $u^{\mathrm{e}}$ satisfies the hypotheses of [15, Lemma 8.1] in the case that $n=3$. Then if $\int_{\Omega} \operatorname{det} \nabla u d x=\int_{\Omega} \operatorname{det} \nabla u_{\lambda} d x$ or if $h^{\prime}\left(\lambda^{3}\right) \leq 0$, the inequality $I(u) \geq I\left(u_{\lambda}\right)$ holds.

Proof By (3.6) it is enough to show that $h^{\prime}\left(\lambda^{3}\right) \int_{\Omega}\left(\operatorname{det} \nabla u-\lambda^{3}\right) d x \geq 0$. The argument which precedes Proposition 2.5 implies that the integral term is not greater than zero, which when coupled with the assumption $h^{\prime}\left(\lambda^{3}\right) \leq 0$ easily gives the desired inequality.

Let $0<\lambda_{1} \leq \lambda_{2} \leq \lambda_{3}$ be the singular values of $\nabla u$ and define the vectors $\Lambda:=$ $\left(\lambda_{1}, \lambda_{2}, \lambda_{3}\right)$ and $\Lambda_{0}:=(\lambda, \lambda, \lambda)$. Recall that

$$
|\nabla u-\lambda \mathbf{1}| \geq\left|\Lambda-\Lambda_{0}\right|
$$

then (3.6) implies

$$
I(u)-I\left(u_{\lambda}\right) \geq \int_{\Omega}\left(\kappa\left|\Lambda-\Lambda_{0}\right|^{q}+\gamma\left|\Lambda-\Lambda_{0}\right|^{2}+h^{\prime}\left(\lambda^{3}\right)\left(\lambda_{1} \lambda_{2} \lambda_{3}-\lambda^{3}\right)\right) d x
$$

The next three results are devoted to the case $h^{\prime}\left(\lambda^{3}\right)>0$.

Lemma 3.2 Let $W$ be as in (3.1) and let $u \in \mathcal{A}_{\lambda}$. Then

$$
I(u)-I\left(u_{\lambda}\right) \geq \int_{\Omega}\left(\mathcal{F}_{1}^{\lambda}(\Lambda)+\mathcal{F}_{2}^{\lambda}(\Lambda)\right) d x,
$$

where

$$
\mathcal{F}_{1}^{\lambda}(\Lambda):=\kappa\left|\Lambda-\Lambda_{0}\right|^{q}+h^{\prime}\left(\lambda^{3}\right)\left(\lambda_{1}-\lambda\right)\left(\lambda_{2}-\lambda\right)\left(\lambda_{3}-\lambda\right)
$$


and

$$
\mathcal{F}_{2}^{\lambda}(\Lambda):=\gamma\left|\Lambda-\Lambda_{0}\right|^{2}+\lambda h^{\prime}\left(\lambda^{3}\right) \sum_{i<j}\left(\lambda_{i}-\lambda\right)\left(\lambda_{j}-\lambda\right) .
$$

Proof For brevity we write $\hat{\lambda}_{i}:=\lambda_{i}-\lambda$ for $i=1,2,3$. It follows that

$$
\operatorname{det} \nabla u-\lambda^{3}=\hat{\lambda}_{1} \hat{\lambda}_{2} \hat{\lambda}_{3}+\lambda \sum_{i<j} \hat{\lambda}_{i} \hat{\lambda}_{j}+\lambda^{2} \sum_{i=1}^{3} \hat{\lambda}_{i}
$$

Inserting this into (3.7) gives

$$
I(u)-I\left(u_{\lambda}\right) \geq \int_{\Omega}\left(\mathcal{F}_{1}^{\lambda}(\Lambda)+\mathcal{F}_{2}^{\lambda}(\Lambda)\right) d x+\lambda^{2} h^{\prime}\left(\lambda^{3}\right) \int_{\Omega} \sum_{i=1}^{3} \hat{\lambda}_{i} d x .
$$

Since the last integral may be written as

$$
\int_{\Omega} \sum_{i=1}^{3} \hat{\lambda}_{i} d x=\int_{\Omega}\left(\lambda_{1}+\lambda_{2}+\lambda_{3}-3 \lambda\right) d x
$$

we can apply [2, Lemma 5.3] again to deduce that

$$
\int_{\Omega}\left(\lambda_{1}+\lambda_{2}+\lambda_{3}\right) d x \geq 3 \lambda \mathcal{L}^{3}(\Omega)
$$

Hence since $h^{\prime}\left(\lambda^{3}\right)>0,(3.8)$ holds.

By analogy with the strategy leading to Lemma 2.9, we now find conditions on $\lambda$ in terms of $\kappa, \gamma$ and $q$ ensuring that

$$
\left\{\begin{array}{l}
\mathcal{F}_{1}^{\lambda}(\Lambda) \geq 0 \\
\mathcal{F}_{2}^{\lambda}(\Lambda) \geq 0
\end{array} \quad \text { for every } \quad \Lambda \in \mathbb{R}^{+++},\right.
$$

where $\mathbb{R}^{+++}:=\left\{x \in \mathbb{R}^{3}: x_{i}>0\right.$ for $\left.i=1,2,3\right\}$.

Lemma 3.3 The functions $\mathcal{F}_{1}^{\lambda}(\Lambda)$ and $\mathcal{F}_{2}^{\lambda}(\Lambda)$ are pointwise nonnegative on $\mathbb{R}^{+++}$provided

$$
\frac{\kappa}{h^{\prime}\left(\lambda^{3}\right) \lambda^{3-q}} \geq(q-2)^{(q-2) / 2} q^{-q / 2}
$$

and

$$
\frac{\gamma}{\lambda h^{\prime}\left(\lambda^{3}\right)} \geq \frac{1}{2}
$$

Proof In the following we let $Y:=h^{\prime}\left(\lambda^{3}\right)>0$ for brevity. We write

$$
\left(\hat{\lambda}_{1}, \hat{\lambda}_{2}, \hat{\lambda}_{3}\right)=\rho(\cos \phi \sin \theta, \sin \phi \sin \theta, \cos \theta),
$$

where $\rho \geq 0$ and $0 \leq \theta \leq \pi, 0 \leq \phi \leq 2 \pi$. In terms of $\rho, \theta$ and $\phi$ we have $\mathcal{F}_{1}^{\lambda}(\Lambda)=$ $F_{1}(\rho, \theta, \phi)$, where

$$
F_{1}(\rho, \theta, \phi):=\kappa \rho^{q}+\frac{Y \rho^{3}}{4} \sin 2 \phi \sin 2 \theta \sin \theta
$$

Since the singular values of $\nabla u$ are ordered as $\lambda_{1} \leq \lambda_{2} \leq \lambda_{3}$ the same applies to the $\hat{\lambda}_{i}$ for $i=1,2,3$; hence in particular $\hat{\lambda}_{1} \leq \hat{\lambda}_{2}$. The latter implies $\phi \in[\pi / 4,5 \pi / 4]$. Now if 
$\sin 2 \phi \cos \theta \geq 0$ then the stated result would be immediate from (3.12). Therefore we assume $\sin 2 \phi \cos \theta<0$ in what follows, which in view of the restriction $\pi / 4 \leq \phi \leq 5 \pi / 4$ implies either that $\phi \in[\pi / 2, \pi]$ when $\cos \theta>0$ or that $\phi \in[\pi / 4, \pi / 2] \cup[\pi, 5 \pi / 4]$ when $\cos \theta<0$. For later use we will let $S$ be the set of $(\theta, \phi)$ satisfying these restrictions.

Let

$$
\bar{\rho}(\theta, \phi):=\inf \left\{\rho>0: F_{1}(\rho, \theta, \phi)=0\right\}
$$

and note that $\bar{\rho}$ is well-defined because, in view of

$$
F_{1}(\rho, \theta, \phi)=\rho^{q}\left(\kappa-\frac{Y \rho^{3-q}}{4}|\sin 2 \phi \sin 2 \theta \sin \theta|\right),
$$

where $q<3$, there is always at least one positive solution to the equation $F_{1}(\rho, \theta, \phi)=0$. Moreover, it is clear that $\bar{\rho}$ satisfies

$$
\frac{4 \kappa}{Y} \bar{\rho}^{q-3}(\theta, \phi)=|\sin 2 \phi \sin 2 \theta \sin \theta| .
$$

Next, let us call $\rho^{*}(\theta, \phi) \geq 0$ an exit radius if

$$
\Lambda_{0}+\rho^{*}(\cos \theta \sin \phi, \sin \theta \sin \phi, \cos \theta) \in \partial \mathbb{R}^{+++} .
$$

Thus $\rho^{*}=\rho_{i}{ }^{*}>0$ for at least one $i$, where

$$
\begin{aligned}
\lambda+\rho_{1}{ }^{*} \sin \theta \cos \phi & =0, \\
\lambda+\rho_{2}{ }^{*} \sin \theta \sin \phi & =0, \\
\lambda+\rho_{3}{ }^{*} \cos \theta & =0 .
\end{aligned}
$$

In order that $\mathcal{F}_{1}^{\lambda}(\Lambda) \geq 0$ for $\Lambda \in \mathbb{R}^{+++}$it should now be clear that $\bar{\rho}$ must exceed the largest exit radius, i.e., $\bar{\rho}(\theta, \phi) \geq \max \left\{\rho_{1}{ }^{*}, \rho_{2}{ }^{*}, \rho_{3}{ }^{*}\right\}$ for each pair $(\theta, \phi)$ in $S$. Rearranging this, we obtain the following sufficient condition:

$$
\frac{4 \kappa}{\lambda^{3-q} Y} \geq \max \left\{s_{1}, s_{2}, s_{3}\right\}
$$

where

$$
\begin{aligned}
& s_{1}:=\sup _{(\theta, \phi) \in S_{1}} \frac{|\sin 2 \phi \sin 2 \theta \sin \theta|}{|\cos \phi \sin \theta|^{3-q}}, \\
& s_{2}:=\sup _{(\theta, \phi) \in S_{2}} \frac{|\sin 2 \phi \sin 2 \theta \sin \theta|}{|\sin \phi \sin \theta|^{3-q}}, \\
& s_{3}:=\sup _{(\theta, \phi) \in S_{3}} \frac{|\sin 2 \phi \sin 2 \theta \sin \theta|}{|\cos \theta|^{3-q}} .
\end{aligned}
$$

Here, $S_{i}=\left\{(\theta, \phi) \in S: \rho_{i}^{*}>0\right\}$ for $i=1,2,3$.

To find $s_{1}$ : Let

$$
m_{1}(\theta, \phi):=4|\sin \phi||\cos \phi|^{q-2}|\cos \theta||\sin \theta|^{q-1},
$$

so that $s_{1}=\max _{S_{1}} m_{1}$. Note that $\rho_{1}{ }^{*}=-\lambda(\cos \phi \sin \theta)^{-1}>0$ implies $\pi / 2<\phi \leq \pi$, which when combined with the restriction $(\theta, \phi) \in S$ implies $\phi \in[\pi / 2, \pi]$ when $\cos \theta>0$ or $\phi \in[\pi, 5 \pi / 4]$ when $\cos \theta<0$. Thus we need only consider these values of $\phi$ when maximizing $m_{1}(\theta, \phi)$ over $S_{1}$. Define $f(\phi):=|\sin \phi||\cos \phi|^{q-2}$ and note that

$$
\max _{S_{1}} m_{1}=4 \max _{0 \leq \theta \leq \pi}|e(\theta)| \max _{[\pi / 2,5 \pi / 4]} f(\phi),
$$


where the function $e$ is defined in (2.29) and its maximum is given by (2.30). Thus

$$
\max _{S_{1}} m_{1}=4(q-1)^{(q-1) / 2} q^{-q / 2} \max _{[\pi / 2,5 \pi / 4]} f(\phi) .
$$

A short calculation shows that $f$ is maximized when $\phi$ satisfies $\cos \phi=-((q-2) /(q-1))^{\frac{1}{2}}$, which is only possible when $\phi$ belongs to $[\pi / 2,3 \pi / 4]$. (It is easy to check that $f$ is monotonic on $[\pi, 5 \pi / 4]$ and that its maximum in this range is smaller than the maximum over the range $[\pi / 2, \pi])$. Hence

$$
\max _{[\pi / 2,5 \pi / 4]} f(\phi)=(q-1)^{\frac{1}{2}}\left(\frac{q-2}{q-1}\right)^{\frac{q-2}{2}},
$$

which gives

$$
\max _{S_{1}} m_{1}=4(q-2)^{(q-2) / 2} q^{-q / 2} .
$$

To find $s_{2}$ : We claim that $s_{2}=s_{1}$. Let

$$
m_{2}(\theta, \phi):=4|\sin \phi|^{q-2}|\cos \phi||\sin \theta|^{q-1}|\cos \theta|
$$

and note that $s_{2}=\max _{S_{2}} m_{2}$. By definition, $(\theta, \phi) \in S_{2}$ are such that $\rho_{2}^{*}>0$, so $\sin \phi<0$, from which (given that $(\theta, \phi) \in S$ ) it follows that $\pi<\phi \leq 5 \pi / 4$. We have $m_{2}(\theta, \phi)=$ $|e(\theta)| \tilde{f}(\phi)$, where the function $e$ was defined in (2.29) and

$$
\tilde{f}(\phi)=|\sin \phi|^{q-2}|\cos \phi| .
$$

It is straightforward to check that the maximum of the function $\tilde{f}$ occurs at $\phi$ such that $\sin \phi=-((q-2) /(q-1))^{\frac{1}{2}}$ and $\cos \phi=-(q-1)^{\frac{1}{2}}$, and that consequently max $\tilde{f}=$ $\max f$, where $f$ is as defined in the previous paragraph. It follows that $s_{2}=s_{1}$.

To find $s_{3}$ : We claim $s_{3}=s_{1}$. Let

$$
m_{3}(\theta, \phi):=2|\sin 2 \phi||\cos \theta|^{q-2} \sin ^{2} \theta
$$

so that $s_{3}=\max _{S_{3}} m_{3}$. Define $r(\theta)=|\cos \theta|^{q-2} \sin ^{2} \theta$. Note that $r$ is symmetric about $\theta=\pi / 2$, so it suffices to consider just its restriction to $[0, \pi / 2]$. A short calculation shows that the maximum of $r$ occurs at $\theta$ satisfying $\sin ^{2} \theta=2 / q$. Thus

$$
\max _{S_{3}} m_{3}=4(q-2)^{(q-2) / 2} q^{-q / 2} .
$$

Condition (3.10) follows by inserting $s_{1}$ into (3.14).

Finally, (3.11) follows by writing $\mathcal{F}_{2}^{\lambda}$ in terms of the coordinates $\Lambda=\Lambda_{0}+\rho\left(l_{1}, l_{2}, l_{3}\right)$ where $l_{1}^{2}+l_{2}^{2}+l_{3}^{2}=1$, giving

$$
\mathcal{F}_{2}^{\lambda}(\Lambda)=\rho^{2}\left(\gamma+\lambda h^{\prime}\left(\lambda^{3}\right)\left(l_{1} l_{2}+l_{1} l_{3}+l_{2} l_{3}\right)\right) .
$$

The minimum of $l_{1} l_{2}+l_{1} l_{3}+l_{2} l_{3}$ among unit vectors $\left(l_{1}, l_{2}, l_{3}\right)$ is $-1 / 2$. Hence $\mathcal{F}_{2}^{\lambda}$ is pointwise nonnegative provided (3.11) holds.

Remark 3.4 It is worth pointing out that the quadratic term in the definition of $W$ cannot be omitted if our method of proof is to work. Nor could this be remedied by considering any adjusted form of $F_{1}, F_{2}$, such as

$$
\widehat{F}_{1}(\rho, \theta, \phi):=\rho^{q} \mu \kappa-\frac{Y \rho^{3}}{4}|\sin 2 \phi \sin 2 \theta \sin \theta|
$$


and

$$
\widehat{F}_{2}(\rho, \theta, \phi):=\rho^{q}(1-\mu) \kappa+\lambda \rho^{2} h^{\prime}\left(\lambda^{3}\right)\left(l_{1} l_{2}+l_{1} l_{3}+l_{2} l_{3}\right),
$$

for some $\mu \in(0,1)$. Indeed, since $q>2$, the first term in $\widehat{F}_{2}$ would be dominated by the term involving $l_{1} l_{2}+l_{1} l_{3}+l_{2} l_{3}$ for sufficiently small $\rho$, and this would prevent the pointwise inequality $\hat{F}_{2} \geq 0$.

The foregoing results imply a three dimensional analogue of Theorem 2.10:

Theorem 3.5 Let the stored energy function $W: \mathbb{R}^{3 \times 3} \rightarrow[0,+\infty]$ be given by

$$
W(A):=|A|^{q}+\gamma|A|^{2}+Z(\operatorname{cof} A)+h(\operatorname{det} A),
$$

where $2<q<3, Z: \mathbb{R}^{3 \times 3} \rightarrow[0,+\infty)$ is convex and $C^{1}$, and $h: \mathbb{R} \rightarrow[0,+\infty]$ satisfies $(H 1)-(H 3)$. Let $\lambda>0$ be such that

$$
\frac{\kappa}{h^{\prime}\left(\lambda^{3}\right) \lambda^{3-q}} \geq(q-2)^{(q-2) / 2} q^{-q / 2}
$$

where $\kappa$ is as per (3.3) and

$$
\frac{\gamma}{\lambda h^{\prime}\left(\lambda^{3}\right)} \geq \frac{1}{2} .
$$

Then any $u \in \mathcal{A}_{\lambda}$ satisfies $I(u) \geq I\left(u_{\lambda}\right)$.

Let us briefly compare the result of Theorem 3.5 with [14, Theorem 4.1]. The latter asserts that under suitable smoothness and convexity assumptions on $h$, a linear deformation $u(x)=L x, u: \Omega \rightarrow \mathbb{R}^{3}$, is a global minimizer of $I$ provided

$$
h^{\prime}(\operatorname{det} L)|L|^{3-q} \leq \frac{c_{1}}{\alpha} .
$$

Here, $\alpha$ and $c_{1}$ are constants which arise in their careful analysis (see [14, Section 3, Remark 2]). Inequalities (3.16) and (3.17) say, in the particular case $L=\lambda \mathbf{1}$, that the affine map $u_{\lambda}$ is a global minimizer of $I$ provided

$$
h^{\prime}(\operatorname{det} L)|L|^{3-q} \leq \min \left\{3^{(3-q) / 2}(q-2)^{(2-q) / 2} q^{q / 2} \kappa, 2\left(3^{(3-q) / 2}\right) \lambda^{2-q} \gamma\right\} .
$$

Thus our result mirrors that of [14] and it produces constants which are explicit up to the inequality (3.3) obeyed by $\kappa$. In fact, ${ }^{5} \kappa$ varies very nearly linearly as a function of $q$ on the interval [2, 3], the approximation $\kappa(q) \sim 3-q+(2-\sqrt{2})(q-2)$ being accurate to within 0.025 for $q$ in $(2,3)$ and exact at the endpoints.

\subsection{Error estimates}

In the three dimensional case error estimates follow an analogous pattern to those given in Sect. 2.1, as we now show. Let $\lambda>0$ be such that

$$
\left\{\begin{array}{l}
\frac{\kappa}{h^{\prime}\left(\lambda^{3}\right) \lambda^{3-q}}>(q-2)^{(q-2) / 2} q^{-q / 2}, \\
\frac{\gamma}{\lambda h^{\prime}\left(\lambda^{3}\right)} \geq \frac{1}{2} .
\end{array}\right.
$$

\footnotetext{
5 This observation is due to Dr. J. Deane, to whom the authors express their gratitude.
} 
Theorem 3.6 Assume that (3.18) holds. Then there is a constant $c=c(\Omega, \lambda, q)>0$ such that for every $u \in \mathcal{A}_{\lambda}$

$$
\int_{\Omega}|\nabla u-\lambda \mathbf{1}|^{q} d x \leq c \delta(u)
$$

where $\delta(u):=I(u)-I\left(u_{\lambda}\right)$.

Proof Throughout this proof $c$ denotes a generic strictly positive constant possibly depending on $\Omega, \lambda$, and $q$.

The second inequality in (3.18) ensures that

$$
\int_{\Omega} \mathcal{F}_{1}^{\lambda}(\Lambda) d x \leq \delta(u) \text { for every } u \in \mathcal{A}_{\lambda},
$$

while the first (strict) inequality in (3.18) yields

$$
\mathcal{F}_{1}^{\lambda}(\Lambda) \geq c\left|\Lambda-\Lambda_{0}\right|^{q} \text { on } \mathbb{R}^{+++},
$$

for some $c>0$. To prove (3.21) we make use of the same notation as in the proof of Lemma 3.3. Let $\epsilon>0$ and observe that

$$
\begin{aligned}
\mathcal{F}_{1}^{\lambda}(\Lambda) & =F_{1}(\rho, \theta, \phi)=\kappa \rho^{q}-\frac{Y \rho^{3}}{4}|\sin 2 \phi \sin 2 \theta \sin \theta| \\
& =(\kappa-\epsilon) \rho^{q}-\frac{Y \rho^{3}}{4}|\sin 2 \phi \sin 2 \theta \sin \theta|+\epsilon \rho^{q} .
\end{aligned}
$$

By applying the reasoning in the proof of Lemma 3.3 to the function

$$
\tilde{F}_{1}(\rho, \theta, \phi):=(\kappa-\epsilon) \rho^{q}-\frac{Y \rho^{3}}{4}|\sin 2 \phi \sin 2 \theta \sin \theta|,
$$

we see that $\tilde{F}_{1} \geq 0$ provided

$$
\frac{\kappa-\epsilon}{\lambda^{q-3} Y} \geq(q-2)^{(q-2) / 2} q^{-q / 2} .
$$

Since $Y:=h^{\prime}\left(\lambda^{3}\right)$, by virtue of the first inequality in (3.18), up to choosing $\epsilon>0$ sufficiently small, (3.22) is clearly fulfilled.

Gathering (3.20), (3.21) and recalling that

$$
\left|\Lambda-\Lambda_{0}\right|=\operatorname{dist}(\nabla u, \lambda S O(3)) \text {, }
$$

we thus obtain

$$
\int_{\Omega} \operatorname{dist}^{q}(\nabla u, \lambda S O(3)) d x \leq c \delta(u) \text { for every } u \in \mathcal{A}_{\lambda} .
$$

Then invoking the rigidity estimate [8, Theorem 3.1] we find $c=c(\Omega)>0$ such that for every $u \in \mathcal{A}_{\lambda}$ there is a constant rotation $R \in S O$ (3) satisfying

$$
\int_{\Omega}|\nabla u-\lambda R|^{q} d x \leq c \delta(u) \text { for every } u \in \mathcal{A}_{\lambda} .
$$

We now claim that

$$
|\mathbf{1}-R|^{q} \leq c \delta(u) .
$$

Combining Jensen's inequality with (3.24) gives

$$
\left(\int_{\Omega}|\nabla u-\lambda R| d x\right)^{q} \leq c \delta(u) .
$$


Set $\tilde{u}:=u / \lambda$ and $\tilde{z}:=\frac{1}{\mathcal{L}^{3}(\Omega)} \int_{\Omega}(\tilde{u}-R x) d x$. Then by Poincaré's inequality together with the continuity of the trace operator we obtain

$$
\int_{\partial \Omega}|\tilde{u}-R x-\tilde{z}| d \mathcal{H}^{2} \leq c \int_{\Omega}|\nabla \tilde{u}-R| d x,
$$

and hence, since $\tilde{u}=x$ on $\partial \Omega$, we deduce

$$
\int_{\partial \Omega}|(\mathbf{1}-R) x-\tilde{z}| d \mathcal{H}^{2} \leq c \int_{\Omega}|\nabla \tilde{u}-R| d x
$$

Arguing as in the proof of [1, Lemma 3.3], we apply [1, Lemma 3.2] to deduce that there exists a universal constant $\sigma>0$ such that

$$
|\mathbf{1}-R| \leq \sigma \min _{z \in \mathbb{R}^{3}} \int_{\partial \Omega}|(\mathbf{1}-R) x-z| d \mathcal{H}^{2} .
$$

Combining (3.26) and (3.27) gives

$$
\begin{aligned}
|\mathbf{1}-R| & \leq c \int_{\Omega}|\nabla \tilde{u}-R| d x \\
& =\frac{c}{\lambda} \int_{\Omega}|\nabla u-\lambda R| d x,
\end{aligned}
$$

and therefore by (3.24) we achieve

$$
|\mathbf{1}-R|^{q} \leq c\left(\int_{\Omega}|\nabla u-\lambda R| d x\right)^{q} \leq c \delta(u),
$$

as claimed.

Finally, choosing $R$ as in (3.24) and combining the latter with (3.28) implies

$$
\begin{aligned}
\int_{\Omega}|\nabla u-\lambda \mathbf{1}|^{q} d x & =\int_{\Omega}|\nabla u-\lambda R+\lambda R-\lambda \mathbf{1}|^{q} d x \\
& \leq c\left(\int_{\Omega}|\nabla u-\lambda R|^{q} d x+\lambda^{q}|\mathbf{1}-R|^{q}\right) \\
& \leq c \delta(u),
\end{aligned}
$$

which is the thesis.

Remark 3.7 If $\lambda$ satisfies (3.18), from (3.19) we can conclude that also in this case $u_{\lambda}$ is the unique global minimiser of $I$ among all maps $u$ in $\mathcal{A}_{\lambda}$ and moreover that $u_{\lambda}$ lies in a potential well.

We end this section by remarking that condition (3.17) can be removed from the statement of Theorem 3.5 if a certain conjecture holds, namely that the function

$$
A \mapsto P(A):=\sum_{i<j} \lambda_{i}(A) \lambda_{j}(A)-\lambda \sum_{i=1}^{3} \lambda_{i}(A)
$$

is quasiconvex at $\lambda \mathbf{1}$. [For $i=1,2,3, \lambda_{i}(A)$ denote, as usual, the singular values of $A \in$ $\left.\mathbb{R}^{3 \times 3}\right]$. Standard results (see, e.g., [6, Theorem 5.39 (ii)]) imply that

$$
A \mapsto \sum_{i<j} \lambda_{i}(A) \lambda_{j}(A)
$$


is polyconvex and hence quasiconvex, but it remains to be seen whether subtracting the term $\sum_{i=1}^{3} \lambda_{i}(A)$ destroys the quasiconvexity at $\lambda \mathbf{1}$. We conjecture that it does not.

To see why the quasiconvexity of $P$ at $\lambda \mathbf{1}$ might matter, note that from (3.9) we can write

$$
\operatorname{det} \nabla u-\lambda^{3}=\hat{\lambda}_{1} \hat{\lambda}_{2} \hat{\lambda}_{3}+\lambda \sum_{i<j} \hat{\lambda}_{i} \hat{\lambda}_{j}+\lambda^{2} \sum_{i=1}^{3} \hat{\lambda}_{i} .
$$

Recalling that $\hat{\lambda}_{i}:=\lambda_{i}-\lambda$ for $i=1,2,3$, where each $\lambda_{i}$ is as before, the quadratic and linear terms in the last line can be expanded and recast as

$$
\lambda \sum_{i<j} \hat{\lambda}_{i} \hat{\lambda}_{j}+\lambda^{2} \sum_{i=1}^{3} \hat{\lambda}_{i}=\lambda \sum_{i<j} \lambda_{i} \lambda_{j}-\lambda^{2} \sum_{i=1}^{3} \lambda_{i},
$$

whose right-hand side we recognise as $\lambda P(\nabla u)$. In summary, we have shown that

$$
\operatorname{det} \nabla u-\lambda^{3}=\hat{\lambda}_{1} \hat{\lambda}_{2} \hat{\lambda}_{3}+\lambda h^{\prime}\left(\lambda^{3}\right) P(\nabla u) .
$$

Inserting this into (3.6) gives (on dropping the term with prefactor $\gamma$, since it will no longer be needed)

$$
\begin{aligned}
I(u)-I\left(u_{\lambda}\right) & \geq \int_{\Omega}\left(\kappa|\nabla u-\lambda \mathbf{1}|^{q}+h^{\prime}\left(\lambda^{3}\right) \hat{\lambda}_{1} \hat{\lambda}_{2} \hat{\lambda}_{3}\right) d x+\lambda h^{\prime}\left(\lambda^{3}\right) \int_{\Omega} P(\nabla u) d x \\
& =\int_{\Omega} \mathcal{F}_{1}^{\lambda}(\Lambda) d x+\lambda h^{\prime}\left(\lambda^{3}\right) \int_{\Omega} P(\nabla u) d x .
\end{aligned}
$$

If $P$ were quasiconvex at $\lambda \mathbf{1}$ then the second integral would by definition satisfy

$$
\int_{\Omega} P(\nabla u) d x \geq \int_{\Omega} P(\lambda \mathbf{1}) d x
$$

for any Lipschitz $u$ which agrees with $u_{\lambda}$ on the boundary of $\Omega$. This, when coupled with a straightforward approximation argument based on the estimate ${ }^{6}$

$$
|P(A)| \leq|A|^{2}+3 \lambda|A|
$$

further implies

$$
\int_{\Omega} P(\nabla u) d x \geq \int_{\Omega} P(\lambda \mathbf{1}) d x
$$

for any $u$ in $W^{1, q}(\Omega)$ with $q \geq 2$. Finally, a short calculation shows that $P(\lambda \mathbf{1})=0$, so that the right-hand side of the last inequality vanishes. Thus the only condition needed in order to conclude that $I(u) \geq I\left(u_{\lambda}\right)$ would be (3.16), which ensures the positivity of the integral involving $\mathcal{F}_{1}^{\lambda}$.

Acknowledgments The authors would like to thank an unknown referee for carefully reading the paper and making a number of useful suggestions.

Open Access This article is distributed under the terms of the Creative Commons Attribution 4.0 International License (http://creativecommons.org/licenses/by/4.0/), which permits unrestricted use, distribution, and reproduction in any medium, provided you give appropriate credit to the original author(s) and the source, provide a link to the Creative Commons license, and indicate if changes were made.

6 This estimate follows from the fact that $A \mapsto \lambda_{i}(A)$ obeys $\lambda_{i}(A) \leq|A|$, which follows easily from the well-known fact that $\sum_{i=1}^{3} \lambda_{i}^{2}(A)=|A|^{2}$. 


\section{References}

1. Agostiniani, V., Dal Maso, G., DeSimone, A.: Linear elasticity obtained from finite elasticity by $\Gamma$ convergence under weak coerciveness conditions. Ann. Inst. H. Poincaré Anal. Non Linéaire. 29, 715-735 (2012)

2. Ball, J.M.: Convexity conditions and existence theorems in nonlinear elasticity. Arch. Rat. Mech. Anal. 63(4), 337-403 (1977)

3. Ball, J.M.: Discontinuous equilibrium solutions and cavitation in nonlinear elasticity. Phil. Trans. R. Soc. Lond. A 306, 557-611 (1982)

4. Ball, J.M., Murat, F.: $W^{1, p}$-quasiconvexity and variational problems for multiple integrals. J. Funct. Anal. 58, 225-253 (1984)

5. Conti, S., Dolzmann, G., Müller, S.: Korn's second inequality and geometric rigidity with mixed growth conditions. Calc. Var. Part. Differ. Equ. 50(1-2), 437-454 (2014)

6. Dacorogna, B.: Direct methods in the calculus of variations. Applied Mathematical Sciences, vol. 78, 2nd edn. Springer, New York (2008)

7. Fonseca, I., Gangbo, W.: Degree theory in analysis and applications. Oxford Lecture Series in Mathematics and its Applications, 2. Oxford Science Publications, The Clarendon Press, Oxford University Press, New York (1995)

8. Friesecke, G., James, R.D., Müller, S.: A theorem on geometric rigidity and the derivation of nonlinear plate theory from three-dimensional elasticity. Comm. Pure Appl. Math. 55, 1461-1506 (2002)

9. Henao, D., Mora-Corral, C.: Invertibility and weak continuity of the determinant for the modelling of cavitation and fracture in nonlinear elasticity. Arch. Rat. Mech. Anal. 197, 619-655 (2010)

10. Henao, D., Mora-Corral, C.: Fracture surfaces and the regularity of inverses for BV deformations. Arch. Rat. Mech. Anal. 201, 575-629 (2011)

11. Müller, S.: Det=det. A remark on the distributional determinant. C. R. Acad. Sci. Paris Sér. I Math. 311, no. 1, 13-17 (1990)

12. Müller, S., Qi, T., Yan, B.S.: On a new class of elastic deformations not allowing for cavitation. Ann. Inst. H. Poincaré Anal. Non Linéaire, 11, no. 2, 217-243 (1994)

13. Müller, S., Palombaro, M.: Derivation of a rod theory for biphase materials with dislocations at the interface. Calc. Var. Part. Differ. Equ. 48(3-4), 315-335 (2013)

14. Müller, S., Sivaloganathan, J., Spector, S.: An isoperimetric estimate and $W^{1, p}$-quasiconvexity in nonlinear elasticity. Calc. Var. Part. Differ. Equ. 8(2), 159-176 (1999)

15. Müller, S., Spector, S.: An existence theory for nonlinear elasticity that allows for cavitation. Arch. Rat. Mech. Anal. 131, 1-66 (1995)

16. Sivaloganathan, J.: Uniqueness of regular and singular equilibria for spherically symmetric problems of nonlinear elasticity. Arch. Rat. Mech. Anal. 96, 97-136 (1986)

17. Sivaloganathan, J., Spector, S.: A construction of infinitely many singular weak solutions to the equations of nonlinear elasticity. Proc. Roy. Soc. Ed. Sect. A 132A, 985-992 (2002)

18. Šverák, V.: Regularity properties of deformations with finite energy. Arch. Rat. Mech. Anal. 100(2), 105-127 (1988)

19. Vodopyanov, S., Gold'shtein, V.: Quasiconformal mappings and spaces of functions with generalized first derivatives. Sib. Math. J. 17, 515-531 (1977)

20. Zhang, K.: Energy minimizers in nonlinear elastostatics and the implicit function theorem. Arch. Rat. Mech. Anal. 114, 95-117 (1991) 